CASE REPORTS

\title{
VESICOUTERINE FISTULA WITH MENOURIA - A CASE REPORT
}

\author{
AKTHER R ${ }^{1}$, SULTANA N ${ }^{2}$, HOSSAIN T $^{3}$, ARA I ${ }^{4}$
}

\begin{abstract}
:
Vesicouterine fistula is a very rare urogenital fistula but may be iatrogenic, following a Caesarean section operation. Presentation and position of fistulas make their diagnoses very difficult. Hysteroscopy, cystoscopy, hysterosalphingography, Computed Tomography scan and Magnetic Resonance Imaging are very useful to diagnose the condition precisely. History of the patient, clinical examination and instillation of methylene blue into the uterine cavity through cervix or through the urethra are an option to diagnose vesicouterine fistula in a low resource centre.
\end{abstract}

Key words: Vesicouterine fistula, Menouria, Caesarean section.

J Dhaka Med Coll. 2010; 19(2) : 150-153.

\section{Introduction:}

Vesicouterine fistula (VUF) is very rare, representing only 1 to $4 \%$ of all cases of urogenital fistula ${ }^{1,2}$. The majority of cases of VUF are iatrogenic, occurring after cesarean section, usually a low segment incision ${ }^{1-4}$. The main symptom of a VUF is urinary incontinence $^{2}$. Other symptoms are cyclic haematuria (menouria), amenorrhoea, and urinary tract infection ${ }^{5}$. The combination of a Caesarean section, amenorrhoea and cyclic hematuria without urinary incontinence has been described as Youssef 's syndrome, which is pathognomonic of a VUF ${ }^{6,7}$. The symptoms may be different and diagnosis may be delayed according to the location and size of fistula, because a functional sphincter is present at the internal uterine orifice ${ }^{8}$. To confirm this fistula imaging study and endoscopy are most useful ${ }^{4}$. A case of vesicouterine fistula can be suspected from history, clinical examination and then assessed by instillation of dye through cervical catheter into uterine cavity and finding it coming out through urethral catheter.

\section{Case Report:}

A 25 years old married woman hailing from Bakergonj, Barisal got admitted into Dhaka Medical College Hospital on 16.01.2010 with the complaints of severe urge incontinence and cyclic incontinence of bloody fluid and/ or urine for 4 to 5 months. According to her statement, she has been married for 8 years. Her first baby was delivered by Caesarean section five years back. During her second delivery, she tried for vaginal delivery at home, later she delivered a dead baby in the hospital by emergency Caesarean section. Despite the presence of intravesicle catheter, she developed incontinence from the $6^{\text {th }}$ post operative day. 4 months later, she reported to the National Fistula Centre, Dhaka Medical College Hospital, Dhaka and diagnosed as a case of ureterovaginal fistula and bilateral ureteric stenting was done. After this treatment, her continuous incontinence changed to severe urge incontinence and cyclical bloody incontinence but she remained dry at night. Physically and mentally, she was sound. Her nervous system and locomotor system was alright. She had no chronic cough, no

1. Dr. Rabeya Akther, Assistant Chief Medical Officer, Bangladesh Bank Medical Center, Dhaka.

2. Dr. Nilufar Sultana, Assistant Professor, Department of Obstetrics \& Gynaecology, Dhaka Medical Collage \& Hospital, Dhaka.

3. Dr. Toufiqua Hossain, Assistant Professor, Department of Obstetrics \& Gynaecology, Dhaka Medical Collage \& Hospital, Dhaka.

4. Dr. Iffat Ara, Associate Professor, Department of Obstetrics \& Gynaecology, Dhaka Medical Collage \& Hospital, Dhaka.

Correspondence: Dr. Rabeya Akther, Assistant Chief Medical Officer, Bangladesh Bank Medical Center, Motijheel, Dhaka, Bangladesh, Tel: 880-2-8313533, Cell Phone: +8801817517100, email: rabeyrakther@yahoo.com 


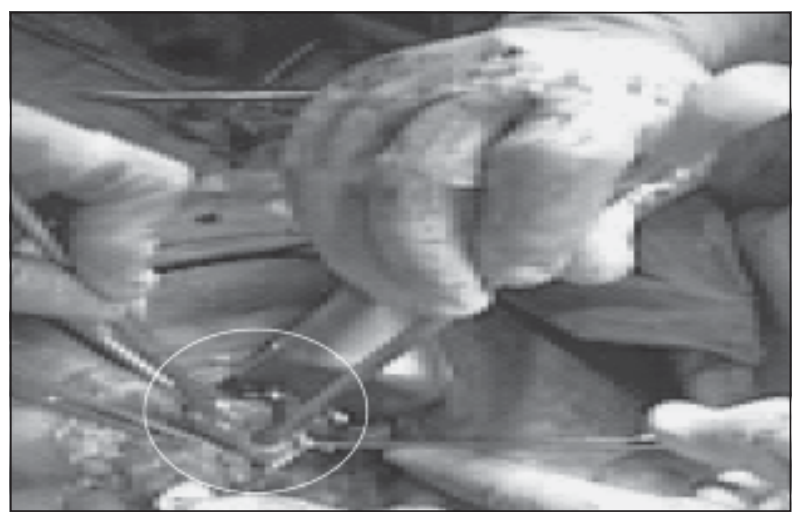

Fig.-1: Detecting the site of the fistula during operation marked by the circle.

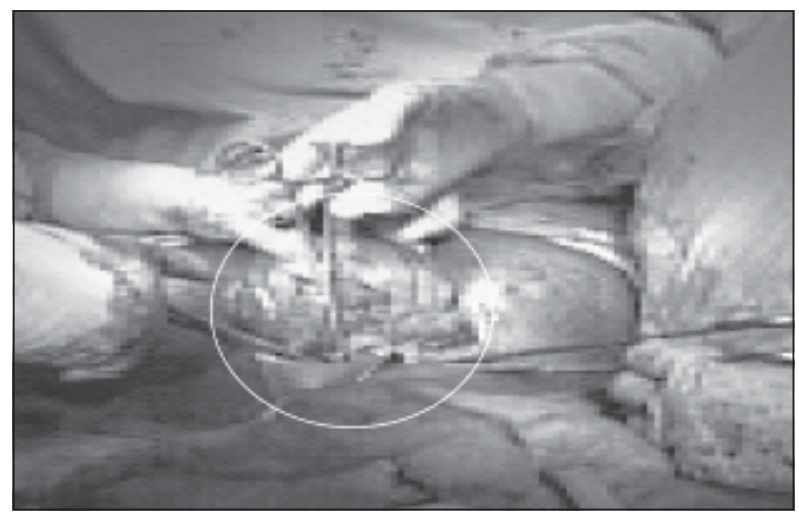

Fig.-2: Repair of the fistula and removal of bilateral ureteric stenting marked by the circle.

constipation, and no history of repeated urinary tract infection. Her stress test was negative, three swab test was positive, vaginoscopy revealed a gap in the lateral fornix. Intravenous urography (IVU) showed normal study. Cystoscopy showed right sided normal ureteric opening and a dilated left one. One opening was found in the left lateral wall of the urinary bladder within a diverticulum. From patient's history, clinical examination and investigations, it was assumed that it was a case of vesico- uterine fistula. After taking all preparation for fistula repair, two catheters were introduced, one in the uterus though cervix and another in the bladder through urethra. Dye was introduced through the uterine catheter and the dye came out through bladder catheter and confirmed the presence of an abnormal tract between urinary bladder and uterine cavity. Abdomen was opened by lower transverse incision and a fistula was found between posterior wall of urinary bladder and lower part of anterior wall of uterus. Mobilization of the posterior bladder wall was done and found to be adherent with the uterus by dense fibrous tissue. There was a single fistula, about $2 \mathrm{~cm} \times 2 \mathrm{~cm}$ in size, just at the level of internal os (Fig. 1,2). The fistulous tract was excised. The bladder and uterus were closed primarily. The bladder was drained through a urethral catheter over 21 days. A drain was in the peritoneal cavity. Since then, urinary and/ or urge incontinence had been stopped.

\section{Discussion:}

Vesicouterine fistula may occur under various conditions, specially after Caesarean section, high forceps' aided delivery, external cephalic version, curettage or manual removal of placenta, placenta percreta, myomectomy, uterine rupture due to obstructed labor, uterine artery embolization, perforation of an intrauterine device, brachytherapy for carcinoma of cervix ${ }^{4}$. Among these, Caesarean section currently is the most common cause of $\mathrm{VUF}^{9}$, and repeated cesarean sections increase the risk of bladder injury and resultant fistulas ${ }^{3}$. In 1957, Youssef described a syndrome comprising of cyclic haematuria, amenorrhoea, menouria and complete urinay incontinence in 6 patients who had lower uterine caesarean section (LUCS). In each case, the symptoms had appeared after an iatrogenic bladder damage, a sinus had resulted which linked the bladder with the uterine cavity, above the level of the internal os ${ }^{6,7}$.

In the present case, the patient had continuous incontinence of urine following Caesarean section. Her incontinence was improved after bilateral ureteric stenting. Her complaints were severe urge incontinence, coughing out of urine and cyclical blood stained incontinence of urine. The clinical presentation is often nonspecific and findings classically used to depict the fistula were negative, leading to considerable delay in diagnosis ${ }^{10}$. The VUF may manifest with continuous urinary incontinence, if the level of the VUF is at or below the level of internal os or the os is incompetent ${ }^{4}$. In this case, 
incontinence of urine occurred more when she felt urge in the presence of a competent os. This may be due to the fact that probably the fistula was large and the contraction of the bladder during micturition caused urine to enter the uterine cavity, through the cervix. Various explanations have been advanced for the mechanism of VUF complicating Cesarean section. Tancer suggested that the most likely mechanism for fistula formation is occult injury to the bladder during low segment cesarean section, such as injury to that portion of the bladder that is sometimes adherent to the anterior vaginal wall or lower segment wall under sharp dissection or the blunt pushing of the bladder downward ${ }^{4}$. Schwartzwald and coworkers suggested that inadequate downward mobilization of the bladder allows for potential injury by an aberrant suture or excessive devascularization, resulting in a $\mathrm{VUF}^{11}$. VUF is often confirmed by instillation of methylene blue in to the uterine cavity or through the urethra or through cervix. But size, site and number of fistula can not be confirmed. To confirm this fistula, imaging study and endoscopy are most useful. In this case, cystoscopy showed a diverticulum within the bladder wall, but failed to confirm the fistula as the tract was long and tortuous ${ }^{6}$. Both the cystoscopy and hysteroscopy have been used in the diagnosis of $\mathrm{VUF}^{9}$. Tancer found that hysteroscopy was the most reliable diagnostic technique ${ }^{4}$. Intravenous urograpy shows the fistula when contrast medium enters the vagina but distinguishing between the vesicovaginal fistula (VVF) and the vesicouterine fistula is difficult. Although VUFs are difficult to diagnose sonographically, sonography shows the fistulous tract as double echogenic lines between the endometrium of the anterior wall of the uterus and mucosal layer of the posterior wall of the bladder ${ }^{9,12}$. But in our case the sonography findings are inconclusive. The ultrasonography has also some inherent difficulty in differentiating UVF tract from different patterns of a noncomplicated Caesarean scar ${ }^{4}$. Magnetic Resonance Imaging (MRI) ${ }^{13}$ and Computed Tomography (CT) $\operatorname{scan}^{14}$ can be used successfully to evaluate both high and low variety of VUF. It gives more information about the precise topography of the fistulous tract and clearly demonstrates the hypo-intense bladder cavity from hyper-intense endometrial cavity due to stagnation of urine. MRI can determine exact position of fistula and surrounding anatomy. It is also a noninvasive method and devoid of contrast media induced nephrotoxicity ${ }^{15}$.

Conclusion: Incontinence of urine due to vesicouterine fistula is difficult to diagnose. Still the availability of high technology diagnostic tests, history and physical examination continue to serve as important initial clinical tools in assessing patient's condition. Intravenous urography and ultrasonography are not very useful for diagnosis. Hysteroscopy, cystoscopy and MRI are appropriate and useful investigation but very costly. For developing countries like Bangladesh, it is difficult to arrange. Instillation of methylene blue into the uterine cavity through cervix or through the urethra helps much in diagnosis and to exclude other possibilities. An accurate history and physical examination can speed up the clinical diagnosis and determine the appropriate therapy without the use of unnecessary and costly tests in low resource centre.

\section{References:}

1. Iloabachie GC, Njoku O. Vesico-uterine fistula. Br J Urol. 1985; 57(4): 438-9.

2. Dubuisson JB, Barbot J, Santarelli J. Vesicouterine fistula after cesarean operation. [Article in French] [Abstract] J Gynecol Obstet Biol Repord. 1979; 8: 229-33.

3. Józwik M, Józwik M, Lotocki W. Vesicouterine fistula: an analysis of 24 cases from Poland. Int J Gynecol Obstet. 1997; 57(2): 169-72.

4. Tancer ML. Vesico-vaginal fistula: a review. Obstet Gynecol Surv. 1986; 41: 743-6.

5. Lenkovsky Z, Pode D, Shapiro A, Caine M. Vesicouterine fistula: a rare complication of caesarean section. J Urol. 1988; 139(1): 123-5.

6. Youssef AF. "Menouria" following lower segment cesarean section: a syndrome. Am J Obstet Gynecol 1957: 73: 759-664.

7. Mori JC. The vesico-vaginal fistula; $2^{\text {nd }}$ ed. London: Bailliere Tindall and Cassell; 1967. p.28. 
8. Wandschneider G. Peritoneal flap plastics in vesicocervical fistula. Int Urol Nephrol. 1986; 18(3): 283-7.

9. Park BK, Kim SH, Cho JY, Sim JS, Seong CK. Vesicouterine fistula after cesarean section: ultrasonographic findings in two cases. J Ultrasound Med. 1999; 18: 441-3.

10. Smayra T, Ghossain MA, Buy JN, Moukarzel M, Jacob D, Truc JB. Vesicouterine fistulas: imaging findings in three cases. Am J Roentgenol. 2005; 184(1): 139-42.

11. Schwartzwald D, Moopan UMM, Tancer ML, Gomez-Leon G, Kim H. Vesicouterine fistula with menouria: a complication from an intrauterine contraceptive device. J Urol. 1986; 136(5): 10667 .
12. Czaplicki M, Go3êtbiewski J, Bablok L, Borkowski A. Diagnosis and treatment of vesicouterine fistula occurring after cesarean section. [Article in Polish] [Abtract] Ginekol Pol. 1997; 68(3): 142-8.

13. Shanmugasundaram R, Gopalakrishnan G, Kekre NS. Youssef's syndrome: is there a better way to diagnose? Indian J Urol. 2008; 24(2): 269-70.

14. Mercader VP, McGuckin JF Jr., Carolin DF, Chatwani A, Seidmon EJ. CT of vesicocorporeal fistula with menouria: a complication of uterine biopsy. J Comput Assist Tomogr. 1995: 19(2): 3246.

15. Murphy JM, Lee G, Sharma SD, Doble A, Lomas DJ. Vesicouterine fistula: MRI diagnosis. Eur Radiol. 1999; 9(9): 1876-8. 